\title{
Alien Mink Predation and Colonisation Processes of Rodent Prey on Small Islands of the Baltic Sea: Does Prey Naïveté Matter?
}

\author{
Karen Fey, ${ }^{1}$ Peter B. Banks, ${ }^{2}$ and Erkki Korpimäki ${ }^{1}$ \\ ${ }^{1}$ Section of Ecology, Department of Biology, University of Turku, 20014 Turku, Finland \\ ${ }^{2}$ School of Biological, Earth and Environmental Sciences, University of New South Wales, Kensington NSW 2052, Australia
}

Correspondence should be addressed to Karen Fey, karen.fey@web.de

Received 17 August 2009; Accepted 24 February 2010

Academic Editor: Andrew Sih

Copyright ( $) 2010$ Karen Fey et al. This is an open access article distributed under the Creative Commons Attribution License, which permits unrestricted use, distribution, and reproduction in any medium, provided the original work is properly cited.

\begin{abstract}
Colonisation, an important part of meta-population dynamics of fragmented populations, depends on both the dispersal ability and the ability to establish in the new habitat. Predation can hinder successful establishment of prey, and where the predation pressure comes from an alien predator, the effects on colonisation might be devastating. We studied the establishment of field voles (Microtus agrestis) inhabiting small islands of the archipelago of the Baltic Sea, SW Finland, under presence and absence of the alien American mink (Mustela vison). We translocated "experienced" voles from islands with mink, and "inexperienced" voles from islands from which mink had been removed, to other islands where mink was present or absent. By radio-tracking we studied survival, space and microhabitat use of voles within four weeks after translocation. Survival of voles on mink islands was significantly lower than on mink-free islands, but "experienced" voles did not survive better than "inexperienced" voles. "Experienced" voles were more often located in juniper habitats than "inexperienced" voles, but they appeared not to gain any survival benefit from altered microhabitat use. This study provides novel evidence, that alien mink predation inhibits establishment of colonising field voles and may thus ultimately induce extinction of voles from the outer archipelago.
\end{abstract}

\section{Introduction}

Many natural populations are fragmented and patchy and thus influenced by metapopulation processes in some form. In true metapopulation dynamics, local populations become extinct, and later on these habitats are recolonized by other individuals [1-3]. Extinctions usually result from extrinsic processes such as overexploitation of limited resources, extirpation by predators, or stochastic catastrophes. In contrast, colonisation is regarded to depend mainly on intrinsic traits of the species, and successful colonisation requires two steps: successful dispersal through the matrix and settling down or establishment in the new habitat after arriving there [4]. In this study we examine how predation from an alien predator can disrupt this colonisation process.

The dispersal process has been well studied so far, but less attention has been paid to factors influencing establishment. It is widely acknowledged that dispersal is dangerous for animals because dispersers often move through unfamiliar or inhospitable terrain, where they may be vulnerable to predation, stress, or malnutrition [5-7]. But once dispersers have arrived, successful colonisation via establishment is also limited. Lack of shelter and unfamiliarity with the habitat may cause intruders to suffer higher mortality than residents [6]. It has also been shown that small mammals are more vulnerable to predation when they are in unfamiliar environments [8]. Intruders therefore should prefer safe, dense microhabitats, that is, habitats offering more shelter and facilities to escape, after arriving at a new patch, especially when predators are present. Where the predation pressure comes from an alien predator, the effects on intruders might be devastating if they lack the appropriate behavioural traits to avoid alien predators [9, 10]. Indeed prey naïvete to the novel predator is often cited as a primary factor in acute vulnerability of native species [11-13]. Because adaptations to minimise predation risk, socalled antipredatory behaviours, typically derive from longterm coevolution between predator and prey, native prey may not be adapted to the hunting strategies of introduced predators [14]. Cox and Lima [9], however, predict that 
such prey naïveté would not be apparent where the invader represents a similar predator archetype to native predator, at the family level. On the other hand, Dickman [11] showed that coevolved avoidance responses can also be lost within a few decades in the absence of predators.

Metapopulation processes, particularly colonisation and extinction, appear to determine the dynamics and distribution of voles in the archipelagos of the Baltic Sea [15]. Both processes are usually related to island size and isolation and are driven by dispersal events, which largely result from overexploitation on small islands [16]. Dispersers leave overgrazed islands and colonise new ones across open water, thus ensuring long-term persistence in the region [15]. But a recently arrived alien predator, the American mink (Mustela vison, hereafter mink), has been shown to have a considerable detrimental impact on the native fauna of the archipelago, including birds, frogs, and small mammals [17-21]. The mink is a semiaquatic species which has continuously escaped from fur farms to North European wetlands for more than 80 years [22]. Twenty years ago mink began to invade the archipelagos of the Baltic Sea [23]. Mink have a diverse diet comprising mainly fish and migratory birds, but they also feed on small rodents and frogs as a supplemental component of their diet $[24,25]$.

American mink in Europe do not necessarily represent a novel predator archetype [9] because there is an assemblage of other mustelid predators across the continent [26]. In the outer archipelago, however, there have been no resident mammalian predators in recent history. Although voles in the archipelago have been shown to recognize the predation risk by mink and to respond, for example, with a microhabitat shift to mink presence [27], vole populations are limited by mink predation during summers with good food availability [19, 28]. Thus, Banks et al. [19] proposed that mink interrupts metapopulation processes in the archipelago by suppressing summer population booms which would otherwise lead to severe overcrowding on the islands and prompt dispersal to maintain colonisation events for the natural metapopulation.

Here we study the effects of mink on colonisation success of voles on small islands of the Baltic Sea. We manipulated mink predation pressure and then translocated "experienced" voles from mink islands and "inexperienced" voles from mink-free islands to other islands where mink was present or absent. Using radio-tracking we studied survival, spacing behaviour, and microhabitat use of the translocated voles. We expected them to survive better on mink free islands, and we expected "experienced" voles to use safer microhabitats and move less than "inexperienced" voles, gaining possibly a survival advantage.

\section{Material and Methods}

2.1. Study Area and Species. Two native vole species are found in the archipelago of the Baltic Sea: the field vole (Microtus agrestis), which is the most common herbivorous small mammal in the archipelago, and the bank vole (Myodes glareolus), which is more patchily distributed and generally more abundant closer to the mainland [29, 30]. For our experiment we chose islands inhabited by field voles only. Field vole densities on the release islands were estimated by setting prebaited traps, using two standard laboratory mouse pellets per trap as bait, for one day. The number of visited traps the next day (estimated from the number of pellets disappeared from the traps, counting one pellet as half a trap) had been shown to be correlated with the number of individuals trapped in another study in the same area [28]. Also Lambin et al. [31] found a linear relationship between vole sign indices and vole density estimates derived from live trapping. We therefore assumed counting visited traps an appropriate estimate of vole population densities and used this method in order not to interfere with the radio-tracking.

Mink is the most important and essentially the only mammalian predator in the study area. Other mammalian predators, such as the red fox (Vulpes vulpes) and the raccoon dog (Nyctereutes procyonoides), are extremely rare on islands of the outer archipelago $[17,18]$, and we did not observe any signs of other mammalian predators than mink during the study. Black adders (Vipera berus) are widespread in North European archipelagos [32] and are regularly observed during summer on all study islands. In addition, during spring and autumn migration, short- and long-eared owls (Asio flammeus and A. otus) and Eurasian kestrels (Falco tinnunculus) probably exert some predation pressure on voles.

The experimental islands were exposed rocky skerries, all $<4$ ha. Vegetation on these islands is sparse and typically dominated by grasses (mainly Festuca spp.), low juniper bushes (Juniperus communis), bog bilberry (Vaccinium uliginosum), and crowberry (Empetrum nigrum) (see [17, 18, 20] for additional details).

2.2. Manipulation of Predation Pressure. Since autumn 1998, mink have been consistently removed by gamekeepers during spring and autumn from 62 islands in a $125 \mathrm{~km}^{2}$ area around Utö near Korppoo $\left(59^{\circ} 47^{\prime} \mathrm{N}, 21^{\circ} 30^{\prime} \mathrm{E}\right)$. A control area of 77 islands across $90 \mathrm{~km}^{2}$ where mink were not removed was established in Vänö near Dragsfjärd $\left(59^{\circ} 48^{\prime} \mathrm{N}, 22^{\circ} 11^{\prime} \mathrm{E}\right)$, another control area consisting of 64 islands across $130 \mathrm{~km}^{2}$ in Brunskär $\left(60^{\circ} 01^{\prime} \mathrm{N}, 21^{\circ} 23^{\prime} \mathrm{E}\right)$ (see $[17,18]$ for methods of removing mink and annual numbers removed, and [19, Figure 1], for a map of the study area).

All removal islands were considered mink-free in summer 2005, whereas mink and signs of mink (fresh scats, kills and prey remains) were observed in the control islands during the same period.

2.3. Vole Tracking. For the experiment we chose five islands of the removal area near Utö, and five islands of the control area near Vänö. Islands were chosen to be as similar as possible in size and vegetation cover to support voles. All experimental islands were inhabited by only one vole species, but vole densities on mink removal islands were naturally higher than those on islands with mink. Voles used in the experiment were trapped on islands not used for translocation in both the mink removal area and the two control areas using Ugglan multiple capture live-traps (Grahnab, Sweden) baited with standard laboratory mouse 
TABLE 1: Number of voles released at the beginning of the experiment and survivors at the end of the experiment.

\begin{tabular}{lcccc}
\hline & \multicolumn{2}{c}{ Beginning of experiment } & \multicolumn{2}{c}{ End of experiment } \\
& Mink free islands & Mink islands & Mink free islands & Mink islands \\
\hline Experienced voles & 4 & 4 & 2 & 0 \\
Inexperienced voles & 6 & 6 & 4 & 1 \\
\hline
\end{tabular}

pellets. Because home range sizes of males and females differ, we concentrated on one sex only. Twenty males were marked with radio-collars (type PIP2, Biotrack, UK) with a mass of $2.0 \mathrm{~g}$. All radio-collared voles were mature with a mean body mass of $38.0 \mathrm{~g}$ (range 26.5-61.0 g). Collars represented ca. $5 \%$ (range $3 \%-7 \%$ ) of body mass of voles. Two males of the same mink experience were released at the same location on each of the experimental islands. In both the mink removal area and the control area, eight "experienced" males from a control area and twelve "inexperienced" males from the mink removal area were released (Table 1). The experiment was conducted during July and early August, a time when most natural dispersal occurs [15].

Radio-tracking started in the morning following the release and was conducted every morning and evening for six days, followed by a break of 14 days and another six days of radio-tracking. Voles were located by triangulation, followed by walking in to obtain the precise location of the tracked vole. Voles were usually located below ground and were not moving when approached; therefore we believe disturbance by the tracking procedure to be low. For each location we recorded the GPS coordinates and the microhabitat type (grassy patches, berries, juniper or open habitats, see [27]) within a $1 \mathrm{~m}$ radius. The expected duration of radiotransmitters was five weeks, and all transmitters were still functioning at the end of the experiment. Voles that were still alive at the end of the experiment were retrapped and their radio-collars were removed.

2.4. Data Analysis. To analyse survival we created KaplanMeier plots and analysed differences in survival distributions using the Breslow-test [33], which is widely used, for example, in medical studies (e.g., [34]) and aimed to identify early losses which is when most mortality during translocations occurs $[35,36]$.

To compare the spacing behaviour and microhabitat use of "experienced" and "inexperienced" voles, we estimated mean interfix distances between consecutive localisations and the mean home range sizes for each vole using the program RANGES 6 [37]. Additionally we calculated the proportion of radio-locations occurring in juniper of all radio-locations, because we found earlier that field voles on mink islands prefer juniper over other habitat types, even though these juniper bushes are also preferred by mink and thus are predicted to be a rather dangerous habitat for voles [27]. In order to test for changes in behaviour during the experimental period we conducted repeated measurement ANOVAs with time after release (week 1 or week 4 after release) as repeated factor and mink presence or absence on both the island of origin and the island of release as factors. Because survival was low, we analysed differences in spacing behaviour and microhabitat use additionally by twoway ANOVA using interfix distances, home range sizes and proportion of radio-locations in juniper during the first week after release as dependent variables, and mink presence or absence on both the island of origin and the island of release as factors.

To analyse whether voles behaving differently gain a survival benefit, we used Spearman's rank correlation and computed correlations between the time span a vole survived (in days) and mobility (mean distances moved and home range sizes during the first week after translocation) and between survival and the proportion of time a vole spent in habitats containing juniper.

Vole densities on the release islands were compared by independent samples $t$-test, using the density index derived from the number of visited traps as test variable and mink absence or presence as grouping variable.

All tests were performed in SPSS 12.0.1 for Windows.

\section{Results}

Seven of the 20 translocated voles survived until the end of the experiment 26 days after the release (Table 1). We found collars of two voles during the experiment. Seven voles which did not move between three consecutive localizations were assumed to be dead. Because collars and locations of nonmoving voles were either in crevices or at the entrance of mink nests, and mink were spotted, or fresh mink droppings were found close-by, we assumed that these voles were killed by mink. Four voles disappeared for unknown reasons, possibly because their radio-transmitters failed or because they were taken by birds of prey and transported far away from the study areas. These voles were excluded from the analyses.

Survival was significantly lower on mink islands than on mink-free islands (Breslow-test, $\chi^{2}=3.95, \mathrm{df}=1, P=$ .047). On mink-free islands, $>50 \%$ of the translocated voles survived until the end of the experiment, whereas on mink islands $50 \%$ of the translocated voles died during the first five days (Figure 1).

The origin of voles did not have an obvious influence on the survival of the translocated voles (Breslow-test, $\chi^{2}=0.01$, $\mathrm{df}=1, P=.925)$. Also on mink islands voles originating from other mink islands did not gain any obvious survival advantage (Breslow-test, $\chi^{2}=1.11$, df $=1, P=.292$ ); that is, they did apparently not benefit from their previous experience with mink.

Distances moved during the first week after release did not differ from the distances moved four weeks after the release (Table 2; repeated measurement ANOVA, $F_{1,7}=$ $0.020, P=.892)$, and home range sizes did not show 


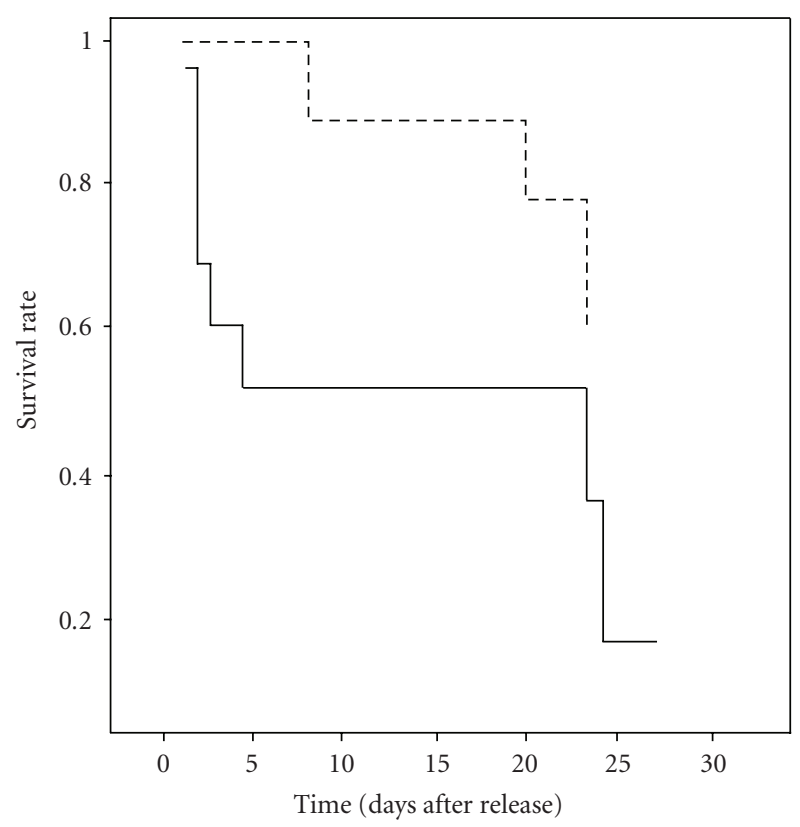

FIGURE 1: The proportion of voles surviving against the cumulative time from release of radio-collared animals on mink (solid line) and mink free (dashed line) islands.

TABLE 2: Mean and SD of distances (in $\mathrm{m}$ ) moved by voles between consecutive localisations during first week and fourth week after translocation depending on the island from which a vole originated (origin) and the island where a vole was released (translocation).

\begin{tabular}{llll}
\hline & & 1st week & 4th week \\
\hline \multirow{2}{*}{ Origin } & Mink island & $12.61 \pm 6.51$ & $16.82 \pm 7.17$ \\
& Mink free island & $11.33 \pm 4.15$ & $7.63 \pm 4.93$ \\
\hline \multirow{2}{*}{ Translocation } & Mink island & $13.99 \pm 4.11$ & $16.41 \pm 10.40$ \\
& Mink free island & $10.93 \pm 5.22$ & $9.12 \pm 5.01$ \\
\hline
\end{tabular}

obvious changes during the experiment (Table 3; repeated measurement ANOVA, $\left.F_{1,7}=4.000, P=.086\right)$. During the first week after release, distances moved between localisations and home range sizes were not influenced by mink presence neither on their original islands nor on the islands where they were transferred to (Table 4). Posthoc power analysis of detectable effect sizes indicates that changes of $67 \%$ in the distance moved and $78 \%$ of home range size were detectable with our sample sizes (using animals on mink free islands as the reference point, assuming $\alpha=0.05, \beta=0.8$ ). Distances moved after translocation appeared not to be higher than distances moved by resident voles on islands in the same area during another experiment (translocated voles: mean $\pm \mathrm{SD}$ $12.7 \pm 9.2 \mathrm{~m}$, resident voles: mean $\pm \mathrm{SD} 17.4 \pm 12.7 \mathrm{~m} ; t$ test, $t=1.351$, $\mathrm{df}=38, P=.185$; Fey et al., unpublished data). Power analysis indicated that differences of greater than $58 \%$ were detectable with our sample size. Mobility of voles was not associated with their survival (Spearman rank correlation, mean distances moved: $N=19, r_{s}=0.186$, $P=.447$, home range sizes: $\left.N=19, r_{s}=0.390, P=.099\right)$.
TABLE 3: Mean and SD of home range sizes (in $\mathrm{m}^{2}$ ) of voles during first week and fourth week after translocation depending on the island from which a vole originated (origin) and the island where a vole was released (translocation).

\begin{tabular}{llll}
\hline & & 1st week & 4th week \\
\hline \multirow{2}{*}{ Origin } & Mink island & $175 \pm 96$ & $125 \pm 50$ \\
& Mink free island & $283 \pm 194$ & $100 \pm 110$ \\
\hline \multirow{2}{*}{ Translocation } & Mink island & $167 \pm 116$ & $100 \pm 0$ \\
& Mink free island & $271 \pm 180$ & $114 \pm 107$ \\
\hline
\end{tabular}

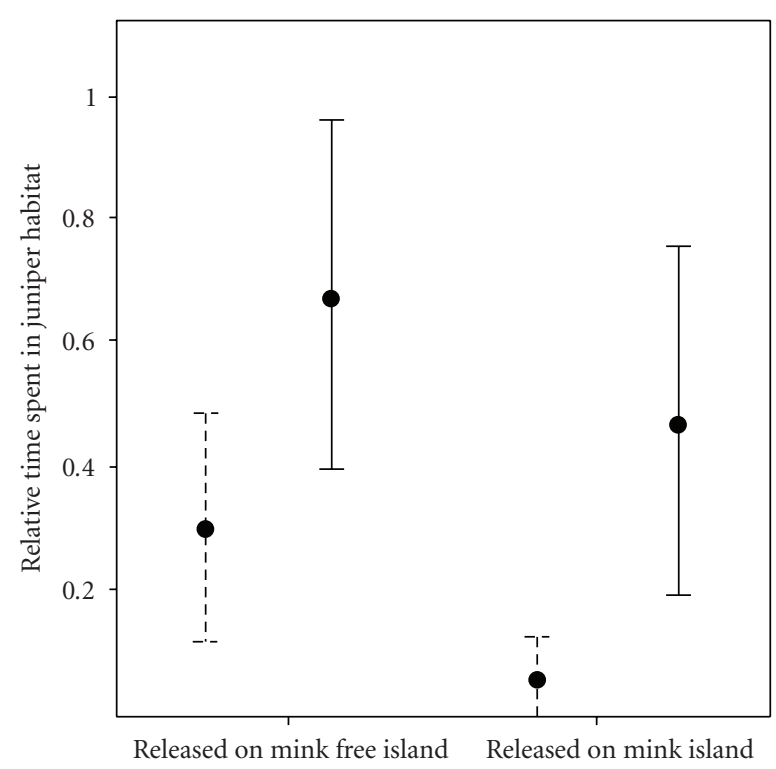

FIGURE 2: The relative time that "experienced" (solid line) and "inexperienced" (dashed line) voles released on mink-free and mink islands spent in juniper in relation to all localisations. Averages and error bars show standard deviations.

There was no difference in the use of juniper habitats between mink and mink-free islands, but "experienced" voles were more often located in juniper than "inexperienced" voles (Figure 2, Table 4). Still, "experienced" voles did not gain a survival benefit from their different habitat use, because there was no obvious correlation between the time span a vole survived (in days) and the proportion of time a vole spent in habitats containing juniper (Spearman rank correlation, $\left.N=20, r_{s}=0.054, P=.820\right)$. Voles did not change their microhabitat use during the experimental period (Table 5; repeated measurement ANOVA, $F_{1,9}=$ $0.416, P=.535)$.

Vole densities on the release islands were higher where mink was absent than where mink was present (mink free islands: mean \pm SD $17.6 \pm 4.6$, mink islands: $10.6 \pm 1.8$; Independent samples $t$-test, $t=2.467, \mathrm{df}=7, t=0.043$ ).

\section{Discussion}

We found a clear negative impact of mink on voles, because survival of translocated voles was dramatically lower on islands where mink was present with half of the voles dying 
TABLE 4: ANOVA on the effects of mink presence or absence on the island from which a vole originated (origin, $\mathrm{O}$ ) and on the island where a vole was released (translocation, $\mathrm{T}$ ) on the space and microhabitat use of voles.

\begin{tabular}{|c|c|c|c|c|c|}
\hline & Source & DF & MS & $F$ & $P$ \\
\hline \multirow{4}{*}{ Distances moved } & Origin & 1 & 0.067 & 0.001 & .979 \\
\hline & Translocation & 1 & 1.723 & 0.019 & .893 \\
\hline & $\mathrm{O} \times \mathrm{T}$ & 1 & 182.978 & 1.972 & .179 \\
\hline & Error & 16 & 92.798 & & \\
\hline \multirow{4}{*}{ Home range sizes } & Origin & 1 & 0.002 & 1.114 & .307 \\
\hline & Translocation & 1 & 0.001 & 0.529 & .478 \\
\hline & $\mathrm{O} \times \mathrm{T}$ & 1 & $<0.001$ & 0.001 & .982 \\
\hline & Error & 16 & 0.002 & & \\
\hline \multirow{4}{*}{ Use of juniper habitats } & Origin & 1 & 0.772 & 6.822 & .019 \\
\hline & Translocation & 1 & 0.136 & 1.198 & .29 \\
\hline & $\mathrm{O} \times \mathrm{T}$ & 1 & 0.022 & 0.196 & .664 \\
\hline & Error & 16 & 0.113 & & \\
\hline
\end{tabular}

TABLE 5: Mean and SD of the proportion (in \%) of localisations of voles in juniper habitats during first week and fourth week after translocation.

\begin{tabular}{llll}
\hline & & 1st week & 4th week \\
\hline Origin & Mink island & $59.0 \pm 39.2$ & $65.4 \pm 40.9$ \\
& Mink free island & $26.4 \pm 32.6$ & $28.3 \pm 36.2$ \\
Translocation & Mink island & $19.5 \pm 32.1$ & $26.3 \pm 32.5$ \\
& Mink free island & $50.3 \pm 37.8$ & $52.5 \pm 44.0$ \\
\hline
\end{tabular}

during the first five days, whereas on mink-free islands more than half of the translocated voles survived until the end of the experiment. Also former experience with mink did not improve survival. Because successful colonisation requires a process of establishment in the new habitat after arrival, our results therefore indicate that alien mink predation impairs the colonisation process of field voles in the archipelago.

Survival of translocated animals is generally low [38, 39]. Poor survival might be related to extensive movements that are often shown after release [40]. However, in our experiment translocated voles appeared to move even shorter distances between radio-locations compared to resident voles on islands in the same area during another experiment, and mobility of voles appeared not to be related to their survival.

Daly et al. [41] stressed the importance of the adverse effects of radio-tags on survival of small rodents. Earlier studies on voles showed, however, that animals' survival was affected by the radio-transmitters only in winter when food availability was low [42]. Since our study was carried out during summer, we feel that the results do not reflect abnormally low survival rates. Moreover, we compared the fate of individuals all equipped with radio-collars. Thus, if radio-collars had an adverse effect, it would have been of the same magnitude on islands with and without mink and for both "inexperienced" and "experienced" voles in both treatments. In addition, Korpimäki et al. [43] found in a study with field voles and their owl predators that radiocollars did not increase the mortality risk of voles. Also
Johannesen et al. [44] did not detect higher mortality in root voles (Microtus oeconomus) wearing radio-collars compared with voles that did not wear collars, and radio-collars have been widely used to study survival rates and mortality factors in voles of the genus Microtus [7, 45-47].

There are three potential reasons for the extremely low survival of translocated field voles on mink islands: (1) voles are naïve to the alien predator and not able to perceive the risk posed by mink (cf. [10], level 1 naïveté), (2) voles perceive the risk but lack the appropriate antipredatory responses against mink (level 2 naïveté), or (3) because of an unfamiliarity with the habitat regardless whether the predator is alien or not $[6,8]$.

Voles on mink and mink free islands appeared not to differ in their space use and microhabitat choice, and they did not change them during the experiment as adaptation to the situation on the island of release. This seems to support the hypothesis that voles in the archipelago are not able to perceive the risk posed by alien mink (level 1 naïveté). Samples sizes were small, which limits the power of our test. However, the detectable shifts in space use (67\% for distance moved, 78\% for home range size) and habitat choice (101\% for juniper use) are within the ranges of observed shifts in behaviour shown by voles to predation risk from natural predators. For example, Korpimäki et al. [43] report a more than sixfold change in use of open habitats by field voles in response to weasel presence. Yet, intrinsic differences in microhabitat use of field voles between "experienced" and "inexperienced" voles suggest that voles are able to perceive the predation risk from alien mink, which might have been facilitated by historic coevolution on the mainland with other mustelids such as weasels and stoats. Mustelids share similarities in hunting techniques and chemical components of their odours which may aid in a general mustelid avoidance strategy [48].

We also found that field voles from islands with mink were more often located in juniper than field voles from mink-free islands, which is consistent with our earlier findings where field voles preferred juniper over other habitat 
types on these small islands [27]. Such responses show that voles are not able to adapt quickly to altered predation risk by mink. Long-term exposure to mink leads to a change in microhabitat use which was also maintained when "experienced" voles were transferred to mink free environments, but which was not induced in "inexperienced" voles by exposure to mink. However, juniper provides probably better shelter against avian predators than against mammalian predators. There is much open space under these bushes, and remains of prey items of mink were often found under juniper (M. Nordström, pers. comm.) during former studies on the islands $[17,18,20]$. Therefore, we assume that mink itself prefers juniper as shelter against predators such as the white-tailed sea eagle (Haliaeetus albicilla), the only native enemy of mink in the archipelago [49]. Thus voles recognised predation risk associated with the alien predator but probably responded in the wrong way (level 2 naïveté).

Establishment in patches already inhabited by conspecifics might be more difficult than recolonisation of empty patches because of intraspecific competition for resources and safe habitats. In our case, however, densities of conspecifics were higher on islands without mink, but still survival and thus establishment was easier on these islands. Thus we conclude that mink predation is the main limiting factor both in colonisation processes of inhabited patches and in recolonisation of empty patches. Because dispersal and recolonisation are essential metapopulation processes, our results support the hypothesis by Banks et al. [19] that alien mink predation may lead to the breakdown of metapopulation dynamics and ultimately cause extinction of the voles in the archipelago.

Our study thus provides the novel evidence that alien predation may inhibit the establishment process of native prey in new habitats. Effective antipredatory behaviour against an alien predator is always critical because of prey naïveté $[9,10]$ but might be even more limited for dispersers [8]. Contrary to residents who are familiar with their habitat and possibly also with the predator, the antipredatory behaviour of dispersers may fail because it is not the appropriate one for that predator in that certain environment. This may lead to failed establishment and thus exaggerate the impact of alien predators.

\section{Acknowledgments}

The authors are grateful to Magnus Perander for boat driving and help with field work and to Pälvi Salo for trapping voles in the mink areas and especially for the emergency takeover of the tracking job. Students of the Ph.D. seminar of the Section of Ecology, University of Turku, gave valuable comments that helped to improve the manuscript. Maj and Tor Nessling Foundation supported the study financially.

\section{References}

[1] R. Levins, "Some demographic and genetic consequences of environmental heterogeneity for biological control," Bulletin of the Entomological Society of America, pp. 15237-15240, 1969.
[2] I. Hanski and M. E. Gilpin, Metapopulation Biology: Ecology, Genetics and Evolution, Academic Press, San Diego, Calif, USA, 1997.

[3] R. Levins, "Extinction," in Lectures on Mathematical Analysis of Biological Phenomena, pp. 123-138, Annals of the New York Academy of Sciences, 1970.

[4] J. A. Stamps, V. V. Krishnan, and M. L. Reid, "Search costs and habitat selection by dispersers," Ecology, vol. 86, no. 2, pp. 510$518,2005$.

[5] P. K. Anderson, "Dispersal in rodents: a resident fitness hypothesis," American Society of Mammalogy Special Publications, pp. 91-141, 1989.

[6] H. Steen, "Low survival of long distance dispersers of the root vole (Microtus oeconomus)," Annales Zoologici Fennici, vol. 31, no. 2, pp. 271-274, 1994.

[7] H. Steen, "Untangling the causes of disappearance from a local population of root voles, Microtus oeconomus: a test of the regional synchrony hypothesis," Oikos, vol. 73, no. 1, pp. 6572, 1995.

[8] L. H. Metzgar, "An experimental comparison of screech owl predation on resident and transient white-footed mice (Peromyscus leucopus)," Journal of Mammalogy, vol. 48, no. 3, pp. 387-391, 1967.

[9] J. G. Cox and S. L. Lima, "Naiveté and an aquatic-terrestrial dichotomy in the effects of introduced predators," Trends in Ecology and Evolution, vol. 21, no. 12, pp. 674-680, 2006.

[10] P. B. Banks and C. R. Dickman, "Alien predation and the effects of multiple levels of prey naïveté," Trends in Ecology and Evolution, vol. 22, no. 5, pp. 229-230, 2007.

[11] C. R. Dickman, "Predation and habitat shift in the house mouse, Mus domesticus," Ecology, vol. 73, no. 1, pp. 313-322, 1992.

[12] P. B. Banks, "Responses of Australian bush rats, Rattus fuscipes, to the odor of introduced Vulpes vulpes," Journal of Mammalogy, vol. 79, no. 4, pp. 1260-1264, 1998.

[13] J. Short, J. E. Kinnear, and A. Robley, "Surplus killing by introduced predators in Australia-evidence for ineffective anti-predator adaptations in native prey species?" Biological Conservation, vol. 103, no. 3, pp. 283-301, 2002.

[14] C. R. Dickman, "Impact of exotic generalist predators on the native fauna of Australia," Wildlife Biology, vol. 2, no. 3, pp. 185-195, 1996.

[15] J. Pokki, "Distribution, demography and dispersal of the field vole, Microtus agrestis (L.), in the Tvärminne archipelago, Finland," Acta Zoologica Fennica, pp. 1641-1648, 1981.

[16] E. E. Crone, D. Doak, and J. Pokki, "Ecological influences on the dynamics of a field vole metapopulation," Ecology, vol. 82, no. 3, pp. 831-843, 2001.

[17] M. Nordström, J. Högmander, J. Nummelin, J. Laine, N. Laanetu, and E. Korpimäki, "Variable responses of waterfowl breeding populations to long-term removal of introduced American mink," Ecography, vol. 25, no. 4, pp. 385-394, 2002.

[18] M. Nordström, J. Högmander, J. Laine, J. Nummelin, N. Laanetu, and E. Korpimäki, "Effects of feral mink removal on seabirds, waders and passerines on small islands in the Baltic Sea," Biological Conservation, vol. 109, no. 3, pp. 359368, 2003.

[19] P. B. Banks, K. Norrdahl, M. Nordström, and E. Korpimäki, "Dynamic impacts of feral mink predation on vole metapopulations in the outer archipelago of the Baltic Sea," Oikos, vol. 105, no. 1, pp. 79-88, 2004.

[20] M. Nordström and E. Korpimäki, "Effects of island isolation and feral mink removal on bird communities on small islands in the Baltic Sea," Journal of Animal Ecology, vol. 73, no. 3, pp. 424-433, 2004. 
[21] M. Ahola, M. Nordström, P. B. Banks, N. Laanetu, and E. Korpimäki, "Alien mink predation induces prolonged declines in archipelago amphibians," Proceedings of the Royal Society of Biological Sciences, vol. 273, no. 1591, pp. 1261-1265, 2006.

[22] N. Dunstone, The Mink, T. \& A. D. Poyser Ltd., London, UK, 1993.

[23] K. Kauhala, "Distributional history of the American mink (Mustela vison) in Finland with special reference to the trends in otter (Lutra lutra) populations," Annales Zoologici Fennici, vol. 33, no. 2, pp. 283-291, 1996.

[24] J. Niemimaa and J. Pokki, "Minkin ravinnosta Suomenlahden ulkosaaristossa," Suomen Riista, pp. 3618-3636, 1990.

[25] P. Salo, M. Toivola, M. Nordström, and E. Korpimäki, "Effects of home range characteristics on the diet composition of female American mink in the Baltic Sea archipelago," Annales Zoologici Fennici, vol. 47, pp. 111-122, 2010.

[26] C. M. King, The Natural History of Weasels and Stoats, Oxford University Press, Oxford, UK, 1989.

[27] K. Fey, P. B. Banks, and E. Korpimäki, "Different microhabitat preferences of field and bank voles under manipulated predation risk from an alien predator," Annales Zoologici Fennici, vol. 43, no. 1, pp. 9-16, 2006.

[28] K. Fey, P. B. Banks, and E. Korpimäki, "Voles on small islands: effects of food limitation and alien predation," Oecologia, vol. 157, no. 3, pp. 419-428, 2008.

[29] E. Kostian, "Habitat requirements and breeding biology of the root vole, Microtus oeconomus (Pallas), on shore meadows inthe Gulf of Bothnia, Finland," Annales Zoologici Fennici, pp. 7329-7340, 1970.

[30] T. Ebenhard, "Demography and island colonization of experimentally introduced and natural vole populations," Acta Universitatis Upsalaiensis, pp. 1411-1428, 1988.

[31] X. Lambin, S. J. Petty, and J. L. Mackinnon, "Cyclic dynamics in field vole populations and generalist predation," Journal of Animal Ecology, vol. 69, no. 1, pp. 106-118, 2000.

[32] A. Forsman and L. E. Lindell, "Responses of a predator to variation in prey abundance: survival and emigration of adders in relation to vole density," Canadian Journal of Zoology, vol. 75, no. 7, pp. 1099-1108, 1997.

[33] N. Breslow, "Covariance analysis of censored survival data," Biometrics, vol. 30, no. 1, pp. 89-99, 1974.

[34] I. Dvorchik and B. I. Carr, "A simple prognostic scoring system for patients with unresectable hepatocellular carcinoma treated by chemo-embolization," Cancer Detection and Prevention, vol. 31, no. 2, pp. 154-160, 2007.

[35] J. Fischer and D. B. Lindenmayer, "An assessment of the published results of animal relocations," Biological Conservation, vol. 96, no. 1, pp. 1-11, 2000.

[36] P. B. Banks, K. Norrdahl, and E. Korpimäki, "Mobility decisions and the predation risks of reintroduction," Biological Conservation, vol. 103, no. 2, pp. 133-138, 2002.

[37] R. E. Kenward, A. B. South, and S. S. Walls, Ranges 6 v1.2: For the Analysis of Tracking and Location Data, Anatrack Ltd., Wareham, UK, 2003.

[38] M. K. O'Bryan and D. R. McCullough, "Survival of blacktailed deer following relocation in California," Journal of Wildlife Management, vol. 49, no. 1, pp. 115-119, 1985.

[39] B. M. Blanchard and R. R. Knight, "Biological consequences of relocating grizzly bears in the Yellowstone ecosystem," Journal of Wildlife Management, vol. 59, no. 3, pp. 560-565, 1995.

[40] D. van Vuren, A. J. Kuenzi, I. Loredo, A. L. Leider, and M. L. Morrison, "Translocation as a nonlethal alternative for managing California ground squirrels," Journal of Wildlife Management, vol. 61, no. 2, pp. 351-359, 1997.
[41] M. Daly, M. I. Wilson, P. R. Behrends, et al., "Sexually differentiated effects of radio transmitters on predation risk and behavior in kangaroo rats Dipodomys merriami," Canadian Journal of Zoology, vol. 70, no. 9, pp. 1851-1855, 1992.

[42] A. B. Webster and R. J. Brooks, "Effects of radiotransmitters on the meadow vole, Microtus pennsylvanicus," Canadian Journal of Zoology, vol. 58, no. 6, pp. 997-1001, 1980.

[43] E. Korpimäki, V. Koivunen, and H. Hakkarainen, "Do radiocollars increase the predation risk of small rodents?" Ethology Ecology and Evolution, vol. 8, no. 4, pp. 377-386, 1996.

[44] E. Johannesen, H. P. Andreassen, and H. Steen, "Effect of radiocollars on survival of root voles," Journal of Mammalogy, vol. 78, no. 2, pp. 638-642, 1997.

[45] K. Norrdahl and E. Korpimäki, "Mortality factors in a cyclic vole population," Proceedings of the Royal Society B, vol. 261, no. 1360, pp. 49-53, 1995.

[46] K. Norrdahl and E. K. Orpimäki, "Does mobility or sex of voles affect risk of predation by mammalian predators?" Ecology, vol. 79, no. 1, pp. 226-232, 1998.

[47] P. B. Banks, K. Norrdahl, and E. Korpimäki, "Nonlinearity in the predation risk of prey mobility," Proceedings of the Royal Society B, vol. 267, no. 1453, pp. 1621-1625, 2000.

[48] D. L. Nolte, J. R. Mason, G. Epple, E. Aronov, and D. L. Campbell, "Why are predator urines aversive to prey?" Journal of Chemical Ecology, vol. 20, no. 7, pp. 1505-1516, 1994.

[49] P. Salo, M. Nordström, R. L. Thomson, and E. Korpimäki, "Risk induced by a native top predator reduces alien mink movements," Journal of Animal Ecology, vol. 77, no. 6, pp. 1092-1098, 2008. 

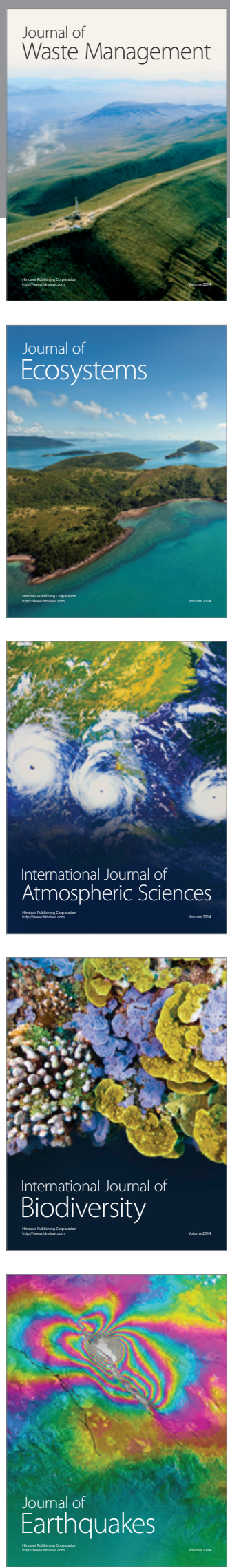
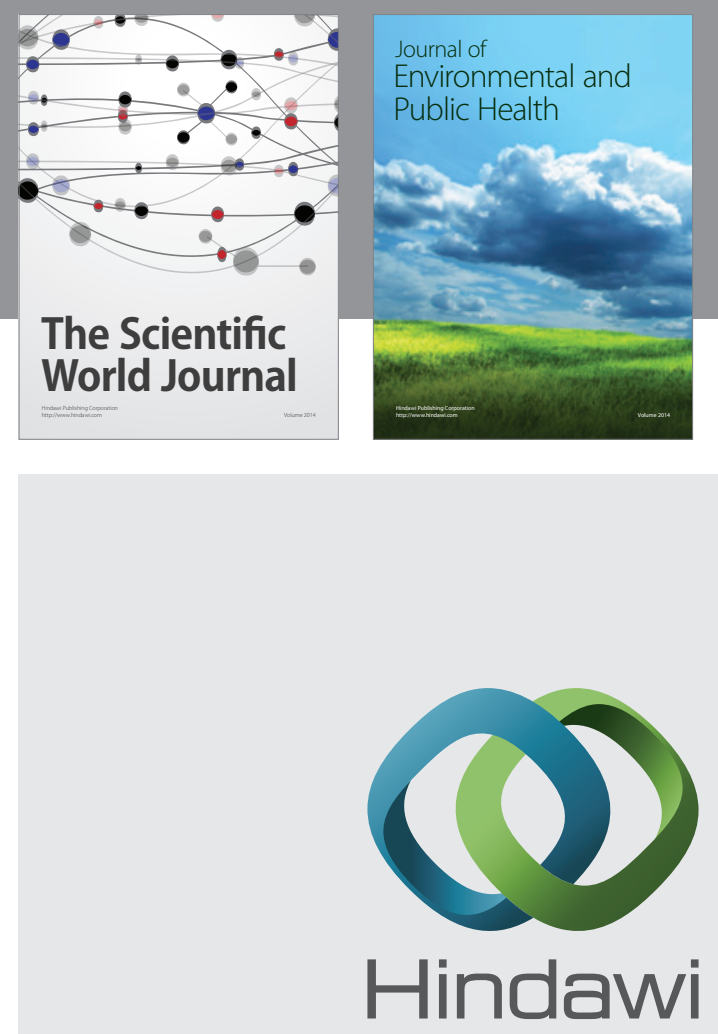

Submit your manuscripts at

http://www.hindawi.com
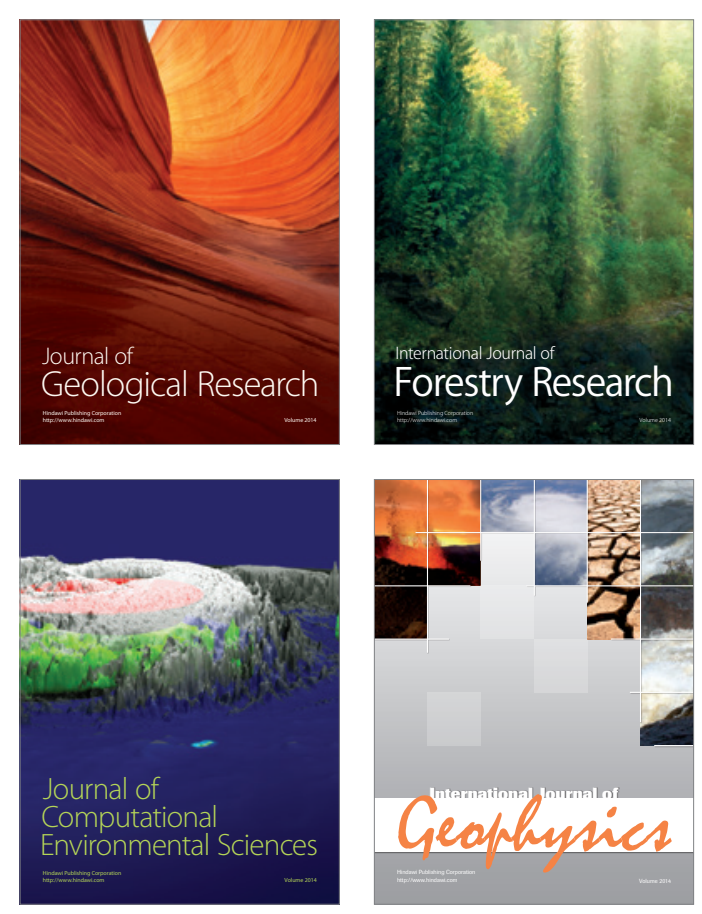
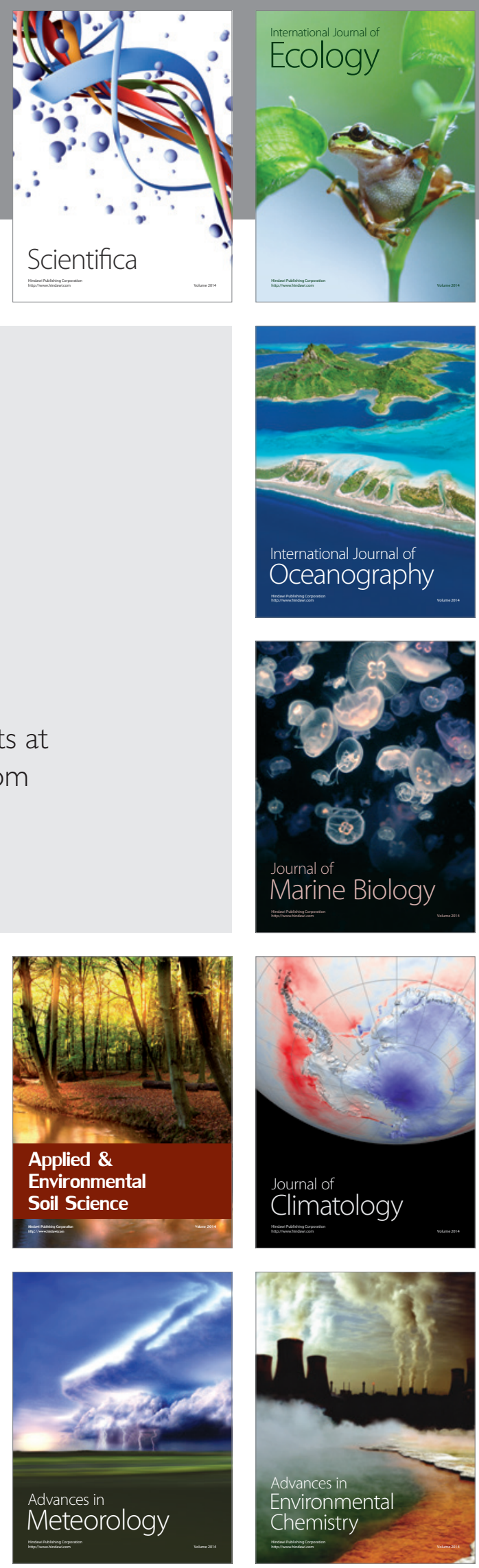